\title{
TEST SEQUENCE SELECTION
}

\author{
David Lee and Ruibing Hao \\ Bell Labs Research China, Beijing, China
}

\begin{abstract}
We study the test sequence selection problem. Given a large number of tests for a protocol system, we want to select a subset of tests so that the number of tests is reduced yet the test coverage is not sacrificed. We discuss the complexity of the test selection problem and propose a class of algorithms for different protocol system information requirements, test coverage criteria, and cost. We report experimental results on ISDN connection control protocol system Q.931.
\end{abstract}

Keywords: protocol system, test sequence, test coverage, extended finite state machine, reachability graph, NP-hard, algorithm, tree, sorting

\section{INTRODUCTION}

With advanced computer technology protocol systems are getting larger to fulfil complicated tasks. However, they are also becoming less reliable. Testing is an indispensable part of system design and implementations; yet it has proved to be a formidable task for complex systems. Because of its practical importance and theoretical interest, there have been a lot of activities on protocol system testing. There are conformance testing, interoperability testing, and performance testing. Conformance testing is to test conformance of system implementations to their specifications [Br, LY1, $\mathrm{PBG}]$. Interoperability testing tends to uncover faults when different system components are interoperating or interfacing with each other [GHLS, KK]. Conformance and interoperability testing are designed to check the correctness of system behaviors whereas performance testing is related to the system performance, such as its transmission rate. 
For conformance testing, test sequences are generated and then applied to the systems under test to conclude whether the implementations conform to their specifications. For the conducted tests to be conclusive, we have to generate test sequences with desired fault coverage. Furthermore, it is costly to execute tests, and we want to minimize the number of tests yet without sacrificing the fault coverage.

For certain complicated legacy protocol systems, such as 5ESS (AT\&T/Lucent No. 5 Electronic Switching System), tests have been generated and applied to the systems over the years at different development stages and by different test engineers. There are thousands of test sequences in the available test set. To test such systems, it is impractical to generate tests from scratch, since it is often impossible to model the systems because they are too complex. Therefore, we want to use the available test set accumulated over years. However, we do not want to execute all of them, since there are too many and to run each test takes a substantial amount of time. A natural solution in practice is to select test sequences among the available test set.

The test selection problem was studied in [LPB] based on the valuations of the test sequences to be selected, and it was reduced to an optimisation problem over Boolean algebra. For testing in context, the problem was studied in [YCL]. An important issue of test selection is the possible loss of coverage. This problem was investigated in [LPB] for partially specified machines and also in $[\mathrm{CV}, \mathrm{VC}, \mathrm{ZV}]$ with an elegant metric of coverage. The selection criteria and their notations were studied in $[\mathrm{Pa}]$.

In this paper, we study the following test selection problem. We have a large set of test sequences, and we want to select a minimal subset of tests to execute without sacrificing the fault coverage. For a formal study, we use extended finite state machine to model protocol systems. It is only for convenience. Our results are easily adaptable to other equivalent mathematical models such as labeled transition systems. We discuss the complexity of the problem and propose efficient algorithms for the test sequence selection.

In Section 2 we describe an extended finite state machine model and its reachability graph. In Section 3 we formulate a problem of test sequence selection, discuss the coverage criteria, and study the problem complexity. In Section 4 we discuss efficient algorithms for the test selection. In Section 5 we discuss a more general problem when the reachability graph is not available. In Section 6 we report experimental results on ISDN Q.931 system 
testing. In Section 7 we conclude the paper with a comparison of all the algorithms studied.

\section{BASICS}

A finite state machine contains a finite number of states and produces outputs on state transitions after receiving inputs. It is often used to model control portions of protocol systems. However, data portions of protocols include variables and operations based on their values; ordinary finite state machines are not powerful enough to model in a succinct way the physical systems any more. Extended finite state machines (EFSM), which are finite state machines extended with variables, have emerged from the design and analysis of communication protocols [LY1]. For instance, IEEE 802.2 LLC [ANSI] is specified by 14 control states, a number of variables, and a set of transitions (pp. 75-117). For a formal definition of EFSM and the related concepts, see [LY1].

Each combination of a state and variable values is called a configuration. An EFSM usually has an initial state $\mathrm{s}_{0}$ and all the variables have an initial value $\mathbf{x}_{\text {init; }}$; we have the initial configuration $\left(s_{0}, \mathbf{x}_{\text {init }}\right)$. A reachability graph consists of all the configurations and transitions, which are reachable from the initial configuration. It is a directed graph where the nodes and edges are the reachable configurations and transitions, respectively. Obviously, a control state may have multiple appearances in the nodes (along with different variable values) and each transition may appear many times as edges in the reachability graph.

For a path from the initial node (configuration) of the reachability graph, the input/output $(\mathrm{I} / \mathrm{O})$ labels on the transitions of the path provide an $\mathrm{I} / \mathrm{O}$ sequence. Conversely, if an I/O sequence corresponds to a unique path from the initial node in the reachability graph, then the underlying EFSM is deterministic. Otherwise, it is non-deterministic. For clarity, in this paper we only consider systems, which are modelled by deterministic EFSM's. Our approaches can be modified to handle non-deterministic EFSM's, as we shall comment in the conclusion of the paper.

Given an input sequence, there is at most one path from the initial node in the reachability graph such that the transitions on the path are labelled with the input sequence, since the machine is deterministic. If such a path exists, then the given input sequence is valid. Otherwise, it is invalid. 
Obviously, a valid input sequence corresponds to a unique I/O sequence, which are the labels on the corresponding unique path from the initial node.

We first study the test selection problem, assuming that the underlying system reachability graph is available. We then relax the problem with an assumption that only the EFSM specification of the underlying system is available. Finally, we present algorithms for a general case when no information of the underlying system is available for test selection.

\section{TEST SEQUENCE SELECTION PROBLEM AND ITS COMPLEXITY}

A test sequence (or a scenario) is valid input sequence of a protocol system that is modelled by an EFSM. Since the system is deterministic, there is a one-to-one correspondence between test sequences and paths in the reachability graph from the initial configuration. In practice, a test sequence usually consists of an I/O sequence; the input sequence is applied to the system under test, and the output sequence is to be observed from the system response. Therefore, a test sequence can be represented by a valid input sequence, a valid I/O sequence, or a path from the initial configuration in the reachability graph of the underlying protocol system. For convenience, we shall use these terms interchangeably.

Informally, the test sequence selection problem is: Given a set of test sequences $\mathrm{S}$, select from $\mathrm{S}$ a subset of tests $\mathrm{S}^{*}$ with a desirable fault coverage. Fault coverage is essential for testing. However, it is often specified differently from different system models and practical needs.

For conformance testing of FSM's we may consider checking sequences [LY1, Mo], which guarantee that an implementation machine is structurally isomorphic to the specification machine. For FSM's there are polynomial time algorithms for constructing the checking sequences, however, even for medium size machines it is too long to be practical [YL1]. For EFSM's hundreds of thousands of states (configurations) are typical and it is in general impossible to construct checking sequences. For the test selection problem, if we are interested in checking sequences, the problem is even harder. Specifically, given a set of tests $S$, we want select a subset $S^{*}$ from $S$ such that $S^{*}$ are checking sequences. The problem is NP-hard [Ya].

A commonly used criterion of fault coverage is to test each edge in the reachability graph at least once. It has been a well-accepted criterion in 
practice, and we first consider this criterion. Formally, given an EFSM M, let $\mathrm{G}$ be its reachability graph with an initial configuration $\mathrm{v}_{0}$, which corresponds to the initial state of $\mathbf{M}$ and the initial variable values. A test sequence of $\mathbf{M}$ is a path in $\mathrm{G}$ from $\mathrm{v}_{0}$. A covering test set is a set of test sequences such that each edge of $G$ is covered by at least one of the test sequences. We want to select a subset of tests such that it is still a covering test set and contains a minimal number of tests:

Problem 1. Test selection with a reachability graph. Given the reachability graph of an EFSM and a covering test set $\mathrm{S}$, select a subset of test sequences $\mathrm{S}^{*}$ from $\mathrm{S}$, such that $\mathrm{S}^{*}$ is a covering test set with a minimal cardinality.

The problem is hard. We can reduce the set cover problem to this test selection problem. Since the set cover problem is NP-hard [GJ], we have:

Proposition 1. The test selection problem 1 is NP-hard.

Therefore, it is hard to obtain an optimal solution for test selection in general. We discuss heuristic methods next.

\section{TEST SEQUENCE SELECTION WITH A REACHABILITY GRAPH}

We consider the following greedy method. We first find a test sequence in $S$ that covers a maximal number of uncovered edges of the reachability graph, and add this test to $S^{*}$. We then repeat the process until all the edges of the reachability graph are covered. Formally, 


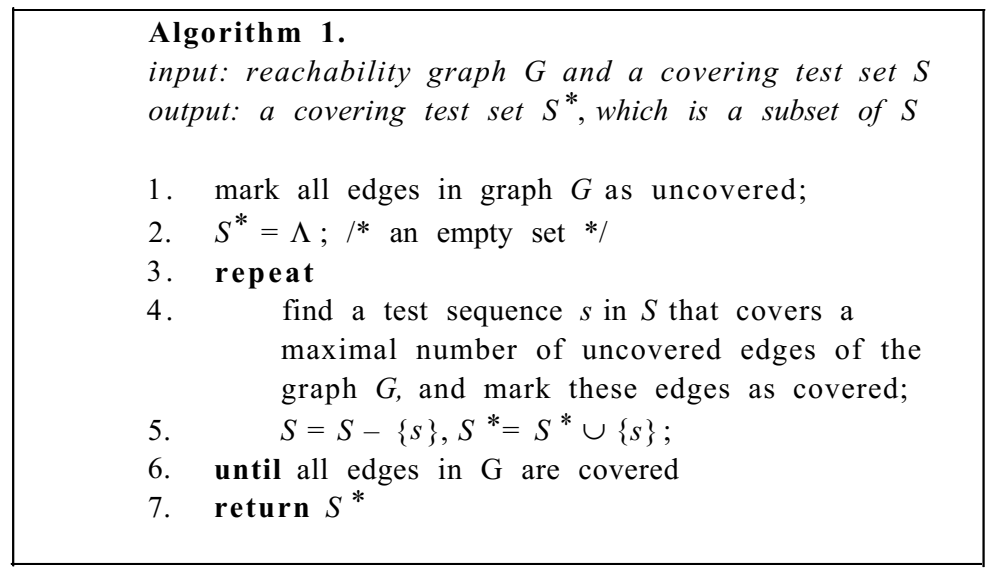

Figure 1. Algorithm 1

It takes polynomial time to find a test sequence $\mathrm{s}$ from $\mathrm{S}$ that covers a maximal number of uncovered edges. A straightforward approach is to examine each test sequence in turn, and it takes time proportional to the length of the test sequence examined. Suppose that there are $\mathrm{N}$ test sequences in $\mathrm{S}$ and the total length of all the test sequences in $\mathrm{S}$ is $\mathrm{M}$. Then it takes time $\mathrm{O}(\mathrm{M})$ to select a test and time $\mathrm{O}(\mathrm{MN})$ in the worst case to determine the test set $\mathrm{S}^{*}$.

Proposition 2. Algorithm 1 takes time $\mathrm{O}(\mathrm{MN})$ to select a covering test set where $\mathrm{M}$ and $\mathrm{N}$ are the total length and number of all the test sequences in the given covering test set.

Algorithm 1 provides a heuristic approach, that is, the selected test set is a covering set but may not be minimal. Instead of selecting one test at a time, we could select two tests at a time such that their combined coverage is maximal [C8]. It increases the cost but still runs in polynomial time. On the other hand, it may reduce the number of selected tests. In general, can we do better than Algorithm 1 in polynomial time? With a similar argument as in [LY2] it can be proved that we cannot do better than a logarithmic factor in polynomial time. That it, for any polynomial time algorithms, which select a covering set of cardinality $\mathrm{N}^{*}$, there are cases such that $\mathrm{N}^{*}=\Omega\left(\mathrm{N}^{* *} \log \mathrm{N}^{* *}\right)$ where $\mathrm{N}^{* *}$ is the cardinality of a minimal covering test set, unless of course $\mathrm{P}=\mathrm{NP}$.

It takes time $\mathrm{O}(\mathrm{MN})$ for Algorithm 1 to select a covering test set where $\mathrm{M}$ and $\mathrm{N}$ are usually large in practice. To reduce the cost we may want to 
avoid examining all the tests in the set. We can conduct the following preprocessing and variations of Algorithm 1.

Algorithm 1.1. We first sort the tests in $\mathrm{S}$ according to their lengths in a descending order, and then examine them in that order.

The rationale is: longer tests correspond to longer paths in the reachability graph and may cover more edges of the graph. Furthermore, if we have found a test, which covers $\mathrm{k}$ uncovered edges, then there is no need to examine tests of length $\mathrm{k}$ or less. Since there are $\mathrm{N}$ tests in the set, the added cost of sorting is $\mathrm{O}(\mathrm{N} \log \mathrm{N})$, which is negligible since it is dominated by $\mathrm{O}(\mathrm{MN})$.

Algorithm 1.2. Select each test sequence to be examined from $S$ uniformly at random.

As often observed in practice, randomization helps. See the experimental results in Section 6.

Algorithm 1.3. Sort the test sequences by their input symbols alphabetically rather than their lengths, remove all the tests which are a proper prefix of other tests, and then apply Algorithm 1.

Obviously, this preprocessing reduces redundant tests and we have fewer tests to process. We shall further discuss this variation in the next section.

\section{TEST SEQUENCE SELECTION WITHOUT A REACHABILITY GRAPH}

Often we only have a test set available but do not have the corresponding reachability graph of the underlying system or it is too costly to construct such a graph. The problem becomes harder since we do not know whether a selected test set covers all the edges in the reachability graph.

Suppose we have the underlying system specification EFSM yet without its reachability graph. We have:

Problem 2. Test selection with an EFSM. Given an EFSM and a covering test set $S$, select a subset of test sequences $S^{*}$ from $S$, such that $S^{*}$ is a covering test set with a minimal cardinality. 
Similar to Problem 1, we have:

Proposition 3. The test selection problem 2 is NP-hard.

Therefore, it is hard to obtain an optimal solution for test selection in general. On the other hand, the remarks on the complexity of Problem 1 also apply to Problem 2.

We can use the following heuristic procedure. We examine all the test sequences in S, trace each sequence in the EFSM, and record all the edges covered in the corresponding reachability graph. We then select test sequences in $\mathrm{S}$ until all the recorded edges are covered. The selection procedure is similar to Algorithm 1, and the variations of Algorithm 1 also apply here. Note that we do not construct the reachability graph explicitly.

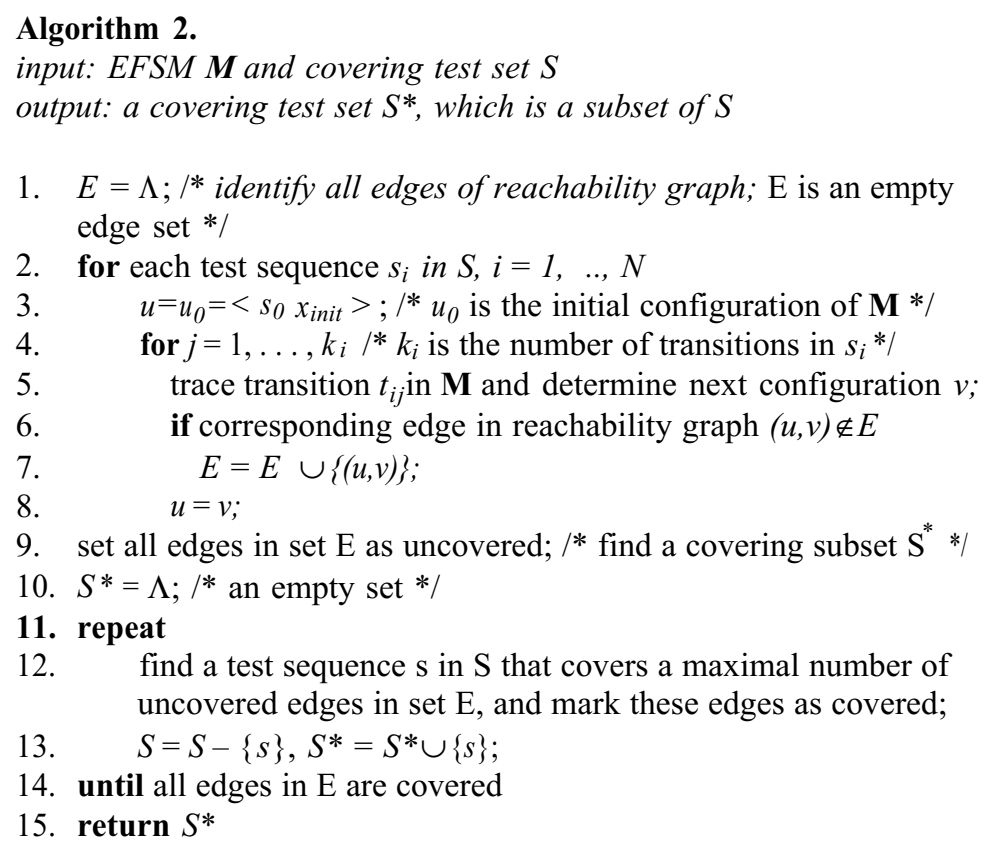

Figure 2. Algorithm 2

Given the underlying EFSM and test set S, Algorithm 2 first collects all the edges of the corresponding reachability graph of the EFSM, and then selects a covering subset of tests. One could construct a reachability graph of 
the EFSM and then select tests using Algorithm 1. However, it is often a difficult task to explicitly construct a reachability graph due to the state explosion. Algorithm 2 avoids this process.

Algorithm 2 traces all the tests in Line 1-8 and records the edges in E. The total number of edges in the worst case can be proportional to $\mathrm{M}$. Therefore, it takes time $\mathrm{O}(\mathrm{M})$ to check if an edge under consideration is in $\mathrm{E}$, and the total time is $\mathrm{O}\left(\mathrm{M}^{2}\right)$. We can keep the recorded edges in set $\mathrm{E}$ in a sorted order (by their inputs, the start and end states and their associated variable values). It takes time proportional to $\mathrm{O}(\log \mathrm{M})$ to check if an edge is in $\mathrm{E}$, and the total time to execute Line $1-8$ is $\mathrm{O}(\mathrm{M} \log \mathrm{M})$. By a same argument as for Algorithm 1, the run time of Line 9-15 is $\mathrm{O}(\mathrm{MN} \log \mathrm{M})$. In summary,

Proposition 4. Algorithm 2 takes time $\mathrm{O}(\mathrm{MNl} \log \mathrm{M})$ to select a covering test set where $\mathrm{M}$ and $\mathrm{N}$ are the total length and number of all the test sequences in the given covering test set.

Algorithm 2 is for a more general test selection Problem 2 where there is no reachability graph and also no need to construct one. However, there is an extra price to pay in terms of the run time, i.e., a factor of $\log M$.

Often in practice the underlying system model may not be available, especially for those legacy systems, and we only have a set of tests to select. This is the most general case of the test selection problem:

Problem 3. Test selection without any information of the underlying protocol system. Given a covering test set $\mathrm{S}$ for a protocol system, for which there is no information available, select a subset of test sequences $S^{*}$, such that $\mathrm{S}^{*}$ is a covering test set.

Different from Problem 1 and 2, in general it is impossible to select a minimal covering test set since there is no information of the underlying protocol system.

For this problem we only consider the following redundancy criterion. Let $\mathrm{s}$ and $\mathrm{s}^{*}$ be two test sequences where $\mathrm{s}^{*}$ is a prefix of $\mathrm{s}$. Let $\mathrm{p}$ and $\mathrm{p}^{*}$ be the corresponding paths from the initial configuration in the reachability graph, which we do not know. Since the corresponding EFSM is deterministic, $\mathrm{p}^{*}$ is a prefix of $\mathrm{p}$ as paths in the reachability graph, and we can remove $\mathrm{s}^{*}$ from the test set without sacrificing the coverage. Consequently, if $\mathrm{S}$ is a covering test set, the test subset $\mathrm{S}^{*}$, selected by discarding all the prefixes, is still a covering test set. As a matter of fact, in 
the worst case, we cannot reduce the tests anymore. Consider the following reachability graph. It consists of separate paths from the initial node. For test selection, we can only discard the paths (tests), which are a proper prefix of another path; any further reduction will lose the coverage.

The above observations lead to the following algorithm. Consider the alphabet set of all the input symbols. We sort all the input sequences in $\mathrm{S}$ alphabetically and then remove all the input sequences (test sequences), which is a prefix of another input sequence. To check whether a test sequence $s_{i}$ is a prefix of another test, there is no need to check $s_{i}$ again all the tests in S; since the test set is sorted, we only need to check $s_{i}$ with the next test sequence $s_{i+1}$

Lemma 1. A Test $s_{i}$ in $\mathrm{S}$ is a prefix of another test if and only if it is a prefix of $s_{i+1}$.

Proof. We only need to prove that if $s_{i}$ is a prefix of another test $s_{i} t$ then it is also a prefix of $s_{i+1}$. Since $s_{i+1}$ is sorted in between $s_{i}$ and $s_{i} t$, it must be of a form $s_{i}$ r. Therefore, $\mathrm{s}_{i}$ is a prefix of $s_{i+1}=s_{i} \quad \mathbf{r}$.

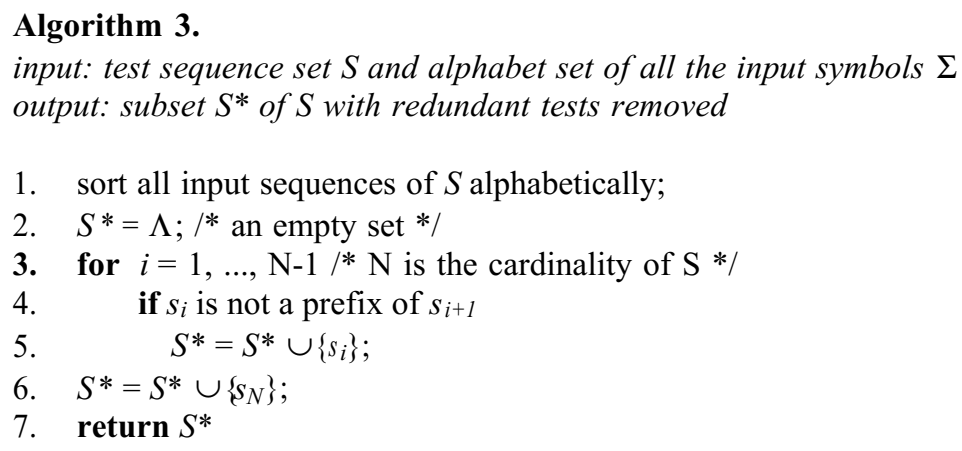

Figure 3. Algorithm 3

Since we only remove the redundant tests, we have:

Proposition 5. Given a test set S, Algorithm 3 removes redundant tests and selects a subset of tests $S^{*}$. If $S$ is a covering test set of the reachability graph of the underlying protocol system, then $\mathrm{S}^{*}$ remains a covering test set.

Note that Algorithm 3 may select test sequences that are redundant in terms of covering the reachability graph since we do not have its information. 
Therefore, the resulting number of tests may be larger than that from Algorithm 1 and 2. See Section 6 for the experimental results.

We now discuss the algorithm complexity. For simplicity, we assume that each test sequence is of length $\mathrm{k}$, i.e., it contains $\mathrm{k}$ input symbols. Suppose that there are $\mathrm{N}$ test sequences in the test set $\mathrm{S}$. Since there are NlogN comparisons to sort $\mathrm{S}$ and each comparison takes time $\mathrm{O}(\mathrm{k})$, it takes time $\mathrm{O}(\mathrm{kN} \log \mathrm{N})$ to sort all the tests in $\mathrm{S}$. We then examine all the tests in the sorted order and remove all the prefixes; the total time is $\mathrm{O}(\mathrm{kN})$. Since the total lengths of all the tests in $\mathrm{S}$ is: $\mathrm{M}=\mathrm{kN}$, we have:

Proposition 6. It takes time $\mathrm{O}(\mathrm{M} \log \mathrm{N})$ to select tests using Algorithm 3 where $\mathrm{M}$ and $\mathrm{N}$ are the total length and number of all the test sequences in the given test set.

The cost of the test selection of Algorithm 3 is dominated by sorting the test sequences. We now discuss another method, using trees. It is optimal in terms of run time; it takes time proportional to the total lengths of the test sequences to be selected.

We grow a tree as follows. Initially, we have a root node $v_{0}$. Each edge is labeled with an input symbol. For each input sequence (test), we walk down the tree as follows. At a node $u$ of the tree with an input symbol $a$ in the sequence, if there is an outgoing edge from $u$ labeled with $a$, then we walk down along that edge, arrive at the end node $v$, and process the input symbol in the test sequence after $a$. However, if there are no outgoing edges labeled with $a$, then we add a tree edge $(u, v)$ from $u$, label it with $a$, walk down along $(u, v)$, and continue processing from $v$ as before. Each test requires a tree walk. After all the tests have been processed, we can obtain the selected tests as follows. We only have to select the tests (or paths) from the root to a leaf node; all the other tests (paths) a prefix of these selected tests. Note that each step of the tree walk takes a constant time (if we have a bitmap at each node to keep track of the labels of all the outgoing edges). Therefore, it takes time proportional to the length of a test sequence to process. Hence the total cost is $\mathrm{O}(\mathrm{M})$.

Proposition 7. It takes time $\mathrm{O}(\mathrm{M})$ to select tests using Algorithm 4 where $\mathrm{M}$ is the total length of all the test sequences in the given test set.

Obviously, Algorithm 4 removes the prefixes and selects the same test set as Algorithm 3. It is more efficient but involves an on-line tree construction process. Again, if the given test set is a covering set then the selected subset 
is also a covering. But it may contain redundant tests in terms of covering the reachability graph, of which we have no information.

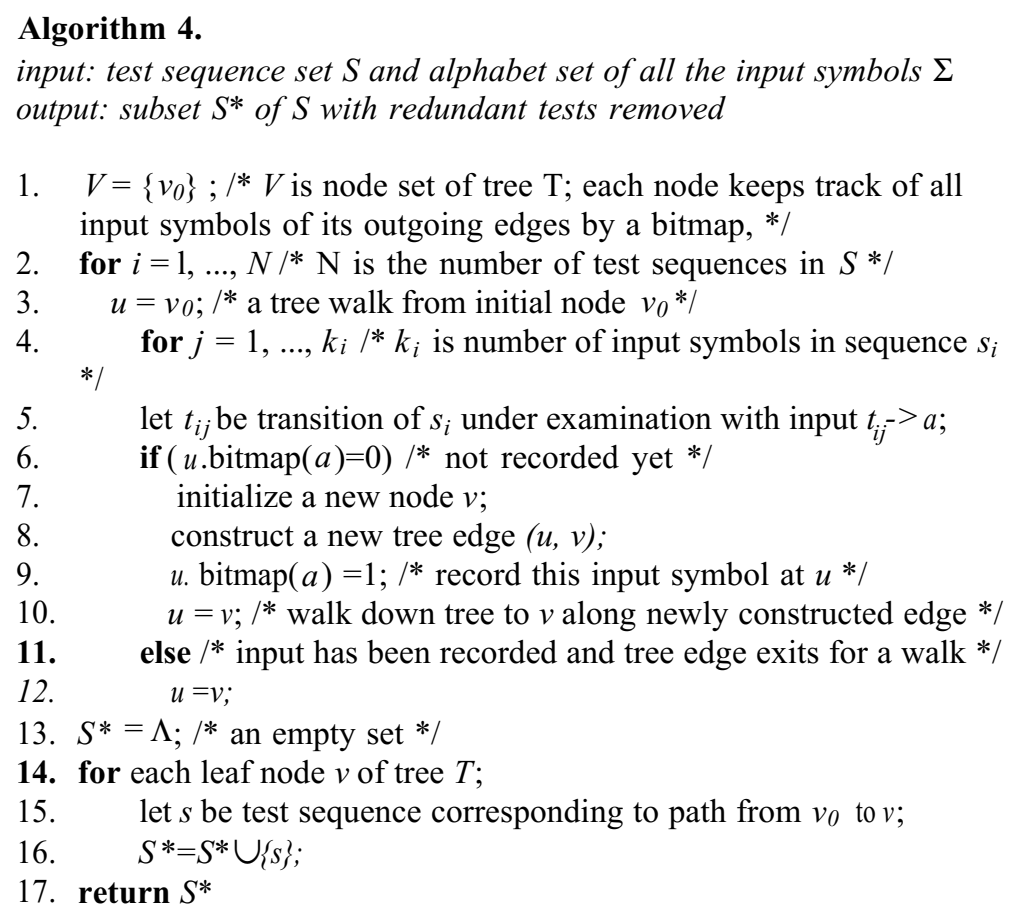

Figure 4. Algorithm 4

\section{EXPERIMENTS}

We applied our test selection algorithms to a simplified version of Q.931 protocol. We now report experimental results. Due to the space limit, for the reachability graph of Q.931 and the test sequences, see [LH].

Q.931 is the connection control protocol for ISDN, comparable to TCP in the Internet protocol stack. It manages connection setup and teardown. However, it does not provide flow control nor perform retransmission, since the underlying layers are assumed to be reliable and the circuit-oriented nature of ISDN allocates bandwidth in fixed increments of $64 \mathrm{kbps}$. Similar to TCP, Q.931 documents both the protocol itself and a protocol state 
machine. For experiment purpose, we constructed an EFSM for Q.931 that covers most of the states and most of the message types. The corresponding reachability graph has 13 nodes and 19 transitions. We also have a set of 59 test sequences for Q.931 that were generated from an automated test generation tool.

We implemented Algorithm 1 and its three variations, and then applied to the 59 test sequence set with the reachability graph. We have:

Table 1. Comparison of Algorithm 1 and its variations

\begin{tabular}{|c|c|}
\hline Algorithms & Number of selected test sequences \\
\hline original test set & 59 \\
\hline Algorithm 1 & 6 \\
\hline Algorithm 1.1 (sort-by-length) & 9 \\
\hline Algorithm 1.2 (random-select) & 8 \\
\hline Algorithm 1.3 (sort-by-alphabet) & 6 \\
\hline
\end{tabular}

From Table 1, all four algorithms reduce the number of final test sequences. According to the discussion in Section 4, Algorithms 1 and 1.3 tend to generate the least number of test sequences. Experimental results in Table 1 verify this observation; both algorithms have selected a set of 6 test sequences, which have the same edge coverage of the reachability graph as the original 59 sequences. Comparing with Algorithm 1 and 1.3, we observe that Algorithm 1.1 and 1.2 execute a little bit faster when processing the same set of input sequences. However, the number of test sequences selected by Algorithm 1.1 and 1.2 is larger than Algorithm 1 and 1.3 (9 and 8 compared to 6); efficiency is achieved with a cost of more redundancy in the tests selected.

We also compare Algorithm 3 and 4 with Algorithm 1 in the number of selected tests and run cost. They use the same set of test sequences as input. The following are the results:

Table 2. Comparison of Algorithm 3, 4 and 1

\begin{tabular}{|c|c|c|c|}
\hline & Algorithm 3 & Algorithm 4 & Algorithm 1 \\
\hline Complexity & $O(M \log N)$ & $O(M)$ & $O(M N)$ \\
\hline number of selected tests & 18 & 18 & 6 \\
\hline
\end{tabular}

From Table 2, Algorithm 3 and 4 both have selected 18 sequences. The run time of Algorithm 4 is less than Algorithm 3. Comparing with Algorithm 1, both Algorithm 3 and 4 selected 12 more test sequences, since Algorithm 3 and 4 do not incorporate the information of the reachability graph of the 
underlying system. On the other hand, the cost of Algorithm 3 and 4 is much less than that of Algorithm 1.

\section{CONCLUSION}

In this paper, we have discussed the test sequence selection problem. Given a test sequence set, we want to select a subset of tests; without sacrificing the coverage we want to minimize the number of the selected tests to reduce the test execution time. We have proposed several algorithms with different required information and coverage, at different costs, and with different redundancy of the selected tests.

Algorithm 1 and its variations Algorithm 1.1-1.3 all require that a reachability graph of the underlying protocol system is available. They are designed for Problem 1 where the coverage is the reachability graph. Algorithm 2 relaxes the requirement; it needs an EFSM of the underlying system. It is designed for Problem 2 where the coverage is also the reachability graph yet only the EFSM of the underlying system is given. Algorithm 3 and 4 are for the most general case when no information is needed for the test selection except for the given test set. They are designed for Problem 3 where there is no information available of the underlying system, and the selected tests also cover the reachability graph if the given test set does.

Algorithm 1 is expensive; it tends to minimize the number of tests, i.e., to reduce the redundancy as much as possible. Algorithm 2 has a same coverage as Algorithm 1, consumes less space (no reachability graph is needed), but takes more time to run. It shows the advantage of using the compact representation EFSM rather than its reachability graph. However, the on-line construction and recording of its edges involve more complicated data structures and algorithms, and it takes longer to run the algorithm. Algorithm 3 costs less than Algorithm 1 and 2. Algorithm 4 is optimal, since its cost is proportional to the total length of tests to be selected; one has to at least read all of them to select.

Algorithm 1 and 2 require more information and is more expensive to run. But they select a smaller test set without sacrificing the coverage. Algorithm 3 and 4 require no information of the underlying system and cost less, but select more tests. Of course they also cover the reachability graph if the given test set does. However, they may contain redundant tests, which are not needed for the coverage of the reachability graph. But there is no way 
one can figure out since there is no information of the underlying protocol system at all.

In practice, often information of the underlying protocol system is not available or it is too expensive to obtain. In this case we resort to Algorithm 3 and 4. As demonstrated by the experiments, they can also reduce significantly the number of tests yet without sacrificing the coverage.

So far we assume that the underlying protocol system is deterministic, and in this case, a valid test sequence corresponds to a unique path in the reachability graph. If the system is non-deterministic, then a valid test sequence may correspond to multiple paths in the graph. Since the execution sequence is non-deterministic, the coverage of the edges in the reachability graph is probabilistic. While the union of all the possible paths, which are associated with a valid test sequence, is considered for coverage, we can only claim in a probabilistic sense.

\section{ACKNOWLEDGEMENTS}

Kun Du from Peking University implemented some of the algorithms. Gerard Holzmann and Margaret Smith from Bell Labs provided us with the set of Ubet scenarios used in our experiments. Our thanks go to the reviewers for their constructive comments and suggestions.

\section{REFERENCES}

[AHU] A.V. Aho, J. E. Hopcroft, and J. D. Ullman, The Design and Analysis of Computer Algorithms, Addison-Wesley, 1974.

[ANSI] International standard ISO 8802-2, ANSI/IEEE std 802.2, 1989.

[Br] E. Brinksma, A Theory for the Derivation of Tests, Proc. PSTV, pp. 63-74, 1988.

[BTV] E. Brinksma, J. Tretmans and L. Verhaard, A Framework for Test Selection, Proc. PSTV, pp. 233-248, 1991.

[C8] Anonymous Reviewer of PSTV-FORTE'2001.

[CV] J. A. Curgus and S. T. Vuong, A Metric Based Theory of Test Selection and Coverage, Proc. PSTV, 1993.

[GJ] M. R. Garey and D. S. Johnson, Computers and Intractability: a Guide to the Theory of NP-Completeness, W. H. Freeman, 1979. 
[GHLS] N. Griffeth, R. Hao, D. Lee, and R. Sinha, Integrated System Interoperability Testing with Applications to VoIP, Proc. FORTE/PSTV, pp. 69-84, 2000.

[KK] S. Kang and M. Kim, Interoperability Test Suite Derivation for Symmetric Communication Protocols, Proc. FORTE/PSTV, 57-72, 1997.

[LH] D. Lee and R. Hao, Test Sequence Selection, Bell Labs Tech. Memo. 2001.

[LY1] D. Lee and M. Yannakakis, Principles and Methods of Testing Finite State Machines a Survey, The Proceedings of IEEE, Vol. 84, No. 8, pp. 1089-1123, 1996.

[LY2] D. Lee and M. Yannakakis, Optimization Problems from Feature Testing of Communication Protocols, Proc. ICNP, pp. 66-75, 1996.

[LPB] G. Luo, A. Petrenko, and G. v. Bochmann, Selecting Test Sequences for Partially Specified Nondeterministci Finite State Machines, Proc. IFIP $7^{\text {th }}$ Int. Workshop on Protocol Test Systems, 1994.

[Mo] E. F. Moore, Gedanken-experiments on sequential machines, Automata Studies, Annals of Mathematics Studies, Princeton University Press, no.34, pp.129-153, 1956.

[Pa] J. Pachl, A Notation for Specifying Test Selection Criteria, Proc. PSTV, pp. 71-84, 1990.

[PY] C. H. Papadimitriou, M. Yannakakis, Optimization, Approximation and Complexity Classes, J. Comp. Sys. Sc., 43(3), 425-440, 1991.

[PBG] A. Petrenko, S. Boroday, R. Groz, Confirming Configurations in EFSM, Proc. FORTE/PSTV, 5-24, 1999.

[VC] S. T. Vuong and J. A. Curgus, On Test Coverage Metrics for Communication Protocols, Proc. IWTCS, 1991.

[Ya] M. Yannakakis, Private communication.

[YCL] N. Yevtushenko, A. Cavalli, L. Lima, Test Suite Minimization for Testing in Context, Proc. IWTCS, pp. 127-145, 1998.

[ZV] J. Zhu and S. T. Vuong, Generalized Metric Based Test Selection and Coverage Measure for Communication Protocols, Proc. PSTV, pp. 299-314, 1997. 